\title{
THE EXISTENCE OF SOLUTIONS FOR A NONLOCAL PROBLEM OF AN IMPLICIT FRACTIONAL-ORDER DIFFERENTIAL EQUATION
}

\author{
Fatma M. Gaafar \\ Faculty of Science, Damanhour University, Damanhour, Egypt*
}

Received 2/8/2017 Revised 28/9/2017 Accepted 1/11/2017

\begin{abstract}
In this paper, we discuss existence of at least one integral solution and also uniqueness for an implicit functional differential equation of fractional order with Riemann-Liouville fractional derivative. The existence will be demonstrated by means of Schauder fixed point theorem and Banach contraction principle. Some examples are also discussed to illustrate the main results.
\end{abstract}

Key words: Riemann-Liouville fractional derivative; Weighted space; Initial value problem; Existence of solutions. Mathematics Subject Classification: 26A33, 34A08, 34A12

\section{Introduction}

Fractional calculus which involves integro differential singular operators has lately caught attracted many engineers, physicists and certainly paying attention mathematician owing to their extensive applications in a multiplicity of fields such as dynamical systems, solid mechanics, viscoelasticity, control etc., see for instance the monographs by: Baleanu et al. [1], Coimbra et al. [2], Coimbra [3], Dalir and Bashour [4], Diethelm [5], Glockle and Nonnenmacher [6], Hilfer [7], Ingman and Suzdalnitsky [8], Kilbas et al. [9], Machado et al. [10], Metzler et al. [11] Rossikhin and Shitikova [12], Sabatier et al. [13], Samko and Marichev [14], Sweilam and AL-Mrawm [15], Yajima and Yamasaki [16] and the references in that.

Numerous researchers form mathematics group fond on investigating existence, stability, uniqueness and additional properties for implicit fractional differential problems (IFDPs) by assorted formulas of fractional differential equations with different formulae of fractional derivative operators. The researchers investigate the case of implicit functions, they considered the nonlinear function $f$ depends on the fractional derivative of the unknown function, see for example, Abbas et al. [17], Benavides [18], Benchohra et al. [19]- [23], El-Sayed and Bin-Taher [24]- [26], Guezane-Lakoud and Khaldi [27], Nieto et al. [28], Vityuk and Mykhailenko [29] and references therein.

The papers on integrable solutions for fractional differential equations is extremely constrained, see papers by: Benchohra et al. [19, 20, 22, 23], El-Sayed and Abd El-Salam [30-32], El-Sayed and Hashem [33] and references therein.

Motivated by the above works, In this paper, we study the existence of at least one integrable solution for the implicit fractional order differential problem (IFDP):

$$
\begin{gathered}
D^{\alpha} u(t)=f\left(t, u(t), D^{\alpha} u(t), \int_{0}^{T} k(t, s) u(s) d s\right) \quad \text { a.e. } t \in(0, T], T<\infty, \\
\left.I^{1-\alpha} u(t)\right|_{t=0}=b \Gamma(\alpha), \quad b \in R,
\end{gathered}
$$

where $f:(0, T] \times R^{3} \rightarrow R, k:[0, T] \times[0, T] \rightarrow R$ are given functions and $D^{\alpha}$ is the Riemann-Liouville fractional-order derivative of order $\alpha \in(0,1)$. Moreover, we will examine the uniqueness of the solution in $L_{1}(J, R), J=(0, T]$ space and in the weighted space $C_{1-\alpha}(J, R)$. We provide examples to clarify our acquired outcomes.

\footnotetext{
*E-mail adress: fatmagaafar2@yahoo.com
} 


\section{Preliminaries}

Let $L_{1}(J, R)$ denoted the space of all Lebesgue integrable functions on the interval $J=(0, T]$ with the standard norm $\|u\|_{L_{1}}=\int_{0}^{T}|u(t)| d t$.

Let $C(J, R)=\left\{u: u(t)\right.$ is continuous on $\left.J:\|u\|=\max _{t \in J}|u(t)|\right\}$,

$C_{1-\alpha}(\bar{J}, R)=\left\{u: t^{1-\alpha} u(t)\right.$ is continuous on $\bar{J}=[0, T]$ with the weighted norm

$$
\left.\|u\|_{C_{1-\alpha}}=\max _{t \in \bar{J}} t^{1-\alpha}|u(t)|\right\} .
$$

Definition 2.1. ( $[1,14])$ The Riemann-Liouville fractional integral of the function $f \in L_{1}(J, R)$ is known as

$$
I^{\alpha} f(t)=\int_{0}^{t} \frac{(t-s)^{\alpha-1}}{\Gamma(\alpha)} f(s) d s, \quad \alpha \in R^{+},
$$

Definition 2.2. ( $[1,14])$ The Riemann-Liouville fractional derivative of $f \in L_{1}(J, R)$ is known as

$$
D^{\alpha} f(t)=\frac{d}{d t} \int_{0}^{t} \frac{(t-s)^{-\alpha}}{\Gamma(1-\alpha)} f(s) d s, \quad \alpha \in(0,1) .
$$

The following theorems will be needed.

Theorem 2.1. (Kolmogorov compactness criterion [34]) Assume that $\Omega \subseteq L^{p}(J, R), 1 \leq p<\infty$. If $\Omega$ is bounded in $L^{p}(J, R)$ and $\lim _{h \rightarrow 0}\left\|u_{h}-u\right\|_{L^{p}}=0$ uniformly for $u \in \Omega$ where

$$
u_{h}(t)=\frac{1}{h} \int_{t}^{t+h} u(s) d s
$$

then in $L_{p}(J, R), \Omega$ is relative compact.

The superposition operator generated by $f$ is identified as follows:

Definition 2.3. [35] Let $f: J \times R \rightarrow R$ be a Carathéodory function. The superposition operator generated by $f(t, x)$ is the operator $F x$ which assigns to each real measurable function on $J$ the real function $(F x)(t)=f(t, x(t)), t \in J$.

Theorem 2.2. (Krasnosel'skii [36]) The superposition operator $F$ created by the function $f$ maps the space $L_{1}(J, R)$ continuously into itself if and only if

$$
|f(t, x)| \leq|a(t)|+b|x|, \quad \text { for all } t \in J \text { and } x \in R,
$$

where $a(t)$ is a function in $L_{1}(J, R)$ and $b$ is a nonnegative constant.

\section{Existence of solutions}

Definition 3.1. A function $u \in L_{1}(J, R)$ is said to be a solution of the IFDP (1.1)-(1.2) if $u$ satisfies (1.1)-(1.2).

Consider the following assumptions

$\left(\mathbf{h}_{\mathbf{1}}\right)$ the function $f: J \times R^{3} \rightarrow R$ is Carathéodory, i.e. measurable in $t \in J$ for each $(u, v, w) \in R^{3}$ and continuous in $(u, v, w) \in R^{3}$ for almost all $t \in J$.

$\left(\mathbf{h}_{\mathbf{2}}\right)$ there is function $a \in L_{1}(J, R)$ and constants $b_{i} \geq 0, i=1,2,3$ such that

$$
|f(t, u, v, w)| \leq|a(t)|+b_{1}|u|+b_{2}|v|+b_{3}|w|, \quad u, v, w \in R \text { and } t \in J .
$$

$\left(\mathbf{h}_{\mathbf{3}}\right) k(t, s)$ is continuous for all $(t, s) \in[0, T] \times[0, T]$ and there is positive constant $K$ such that $\max _{t, s \in[0, T]}|k(t, s)| \leq K$.

For the existence of a solution to problem (1.1)-(1.2), we need to the following lemma.

Lemma 3.1. The problem

$$
D^{\alpha} u(t)=y(t),
$$

under the condition $\left.\quad I^{1-\alpha} u(t)\right|_{t=0}=b \Gamma(\alpha)$, 
is equivalence to the Volterra integral equation

$$
u(t)=b t^{\alpha-1}+\int_{0}^{t} \frac{(t-s)^{\alpha-1}}{\Gamma(\alpha)} y(s) d s .
$$

Proof. Letting $D^{\alpha} u(t)=y(t)$, then $D I^{1-\alpha} u(t)=y(t)$, integrating both sides from 0 to $t$, we get

$$
\begin{gathered}
I^{1-\alpha} u(t)-\left.I^{1-\alpha} u(t)\right|_{t=0}=\int_{0}^{t} y(s) d s, \\
I^{1-\alpha} u(t)=b \Gamma(\alpha)+\int_{0}^{t} y(s) d s,
\end{gathered}
$$

operating by $I^{\alpha}$ on both sides, we get

$$
I u(t)=\frac{b t^{\alpha}}{\alpha}+I^{\alpha+1} y(t)
$$

differentiating both sides, we get (3.1).

Conversely, let $u(t)$ be a solution of (3.1), operating by $I^{1-\alpha}$ on it, then

$$
\begin{aligned}
I^{1-\alpha} u(t) & =I^{1-\alpha} b t^{\alpha-1}+I^{1-\alpha} \int_{0}^{t} \frac{(t-s)^{\alpha-1}}{\Gamma(\alpha)} y(s) d s, \\
& =b \Gamma(\alpha)+\int_{0}^{t} y(s) d s, \\
\text { and }\left.\quad I^{1-\alpha} u(t)\right|_{t=0} & =b \Gamma(\alpha) .
\end{aligned}
$$

Lemma 3.2. The solution of the IFDP (1.1)-(1.2) if it exists, it has the integral form

$$
u(t)=b t^{\alpha-1}+\int_{0}^{t} \frac{(t-s)^{\alpha-1}}{\Gamma(\alpha)} y(s) d s
$$

where $y(t)$ is the solution of the functional integral equation

$$
y(t)=f\left(t, b t^{\alpha-1}+I^{\alpha} y(t), y(t), b \int_{0}^{T} s^{\alpha-1} k(t, s) d s+\int_{0}^{T} k(t, s) \int_{0}^{s} \frac{(s-\xi)^{\alpha-1}}{\Gamma(\alpha)} y(\xi) d \xi d s\right)
$$

Proof. Letting $y(t)$ be such that $y(t)=D^{\alpha} u(t)$, substituting in equation (1.1), then we have

$$
y(t)=f\left(t, u(t), y(t), \int_{0}^{T} k(t, s) u(s) d s\right)
$$

using Lemma 3.1,

$$
u(t)=b t^{\alpha-1}+\int_{0}^{t} \frac{(t-s)^{\alpha-1}}{\Gamma(\alpha)} y(s) d s
$$

substitute by the estimation of $u(t)$ in (3.4), then we get (3.3).

Theorem 3.1. Let the assumptions $\left(h_{1}\right)-\left(h_{3}\right)$ are satisfied.

$$
\text { If } \frac{b_{1} T^{\alpha}}{\Gamma(\alpha+1)}+b_{2}+\frac{b_{3} K T^{\alpha+1}}{\Gamma(\alpha+1)}<1,
$$

then IFDP (1.1)-(1.2) has at least one solution $u \in L_{1}(J, R)$.

Proof. Convert functional integral equation (3.3) into a fixed point problem. Consider the following operator $F$

$$
F: L_{1}(J, R) \rightarrow L_{1}(J, R)
$$

defined by

$$
(F y)(t)=f\left(t, b t^{\alpha-1}+I^{\alpha} y(t), y(t), b \int_{0}^{T} s^{\alpha-1} k(t, s) d s+\int_{0}^{T} k(t, s) \int_{0}^{s} \frac{(s-\xi)^{\alpha-1}}{\Gamma(\alpha)} y(\xi) d \xi d s\right) .
$$


Let $B_{r}=\left\{y \in L_{1}(J, R):\|y\|_{L_{1}} \leq r\right\} \subset L_{1}(J, R)$, where

$$
r \geq \frac{\|a\|_{L_{1}}+\frac{b_{1}|b|}{\alpha} T^{\alpha}+\frac{b_{3}|b| K T^{\alpha+1}}{\alpha}}{1-\left(\frac{b_{1} T^{\alpha}}{\Gamma(\alpha+1)}+b_{2}+\frac{b_{3} K T^{\alpha+1}}{\Gamma(\alpha+1)}\right)} .
$$

Evidently, $B_{r}$ is closed, bounded and also convex.

Now, we will demonstrate that $F B_{r} \subset B_{r}$; actually, from (3.5), (3.6) and from the assumptions $\left(h_{2}\right)-\left(h_{3}\right)$, let $y$ be an arbitrary element in $B_{r}$, we have

$$
\begin{aligned}
& \|F y\|_{L_{1}}=\int_{0}^{T}|(F y)(t)| d t \\
= & \int_{0}^{T}\left|f\left(t, b t^{\alpha-1}+I^{\alpha} y(t), y(t), b \int_{0}^{T} s^{\alpha-1} k(t, s) d s+\int_{0}^{T} k(t, s) \int_{0}^{s} \frac{(s-\xi)^{\alpha-1}}{\Gamma(\alpha)} y(\xi) d \xi d s\right)\right| d t, \\
\leq & \int_{0}^{T}\left(|a(t)|+b_{1}|b| t^{\alpha-1}+b_{1} \int_{0}^{t} \frac{(t-s)^{\alpha-1}}{\Gamma(\alpha)}|y(s)| d s+b_{2}|y(t)|+b_{3}|b| \int_{0}^{T} s^{\alpha-1}|k(t, s)| d s\right. \\
& \left.+b_{3} \int_{0}^{T}|k(t, s)| \int_{0}^{s} \frac{(s-\xi)^{\alpha-1}}{\Gamma(\alpha)}|y(\xi)| d \xi d s\right) d t \\
\leq & \|a\|_{L_{1}}+\frac{b_{1}|b| T^{\alpha}}{\alpha}+b_{1} \int_{0}^{T} \int_{0}^{t} \frac{(t-s)^{\alpha-1}}{\Gamma(\alpha)}|y(s)| d s d t+b_{2}|| y \|_{L_{1}} \\
& +b_{3}|b| \int_{0}^{T} \int_{0}^{T} s^{\alpha-1}|k(t, s)| d s d t+b_{3} K \int_{0}^{T} \int_{0}^{T} \int_{0}^{s} \frac{(s-\xi)^{\alpha-1}}{\Gamma(\alpha)}|y(\xi)| d \xi d s d t \\
\leq & \|a\|_{L_{1}}+\frac{b_{1}|b| T^{\alpha}}{\alpha}+b_{1} \int_{0}^{T}\left|y(s) d s \int_{s}^{T} \frac{(t-s)^{\alpha-1}}{\Gamma(\alpha)} d t+b_{2}\right| \mid y \|_{L_{1}} \\
& +b_{3}|b| K \int_{0}^{T} \frac{T^{\alpha}}{\alpha} d t+b_{3} K \int_{0}^{T} d t \int_{0}^{T}|y(\xi)| d \xi \int_{\xi}^{T} \frac{(s-\xi)^{\alpha-1}}{\Gamma(\alpha)} d s, \\
\leq & \|a\|_{L_{1}}+\frac{b_{1}|b| T^{\alpha}}{\alpha}+\frac{b_{1} T^{\alpha}}{\Gamma(\alpha+1)}\|y\|_{L_{1}}+b_{2}\|y\|_{L_{1}}+\frac{b_{3}|b| K T^{\alpha+1}}{\alpha} \\
& +b_{3} K \int_{0}^{T} \int_{0}^{T} \frac{(T-\xi)^{\alpha}}{\Gamma(\alpha+1)}|y(\xi)| d \xi d t \\
\leq & \|a\|_{L_{1}}+\frac{b_{1}|b| T^{\alpha}}{\alpha}+\frac{b_{1} T^{\alpha}}{\Gamma(\alpha+1)}\|y\|_{L_{1}}+b_{2}\|y\|_{L_{1}}+\frac{b_{3}|b| K T^{\alpha+1}}{\alpha}+\frac{b_{3} K T^{\alpha+1}}{\Gamma(\alpha+1)}\|y\|_{L_{1}}, \\
\leq & \|a\|_{L_{1}}+\frac{b_{1}|b| T^{\alpha}}{\alpha}+\frac{b_{1} T^{\alpha}}{\Gamma(\alpha+1)} r+b_{2} r+\frac{b_{3}|b| K T^{\alpha+1}}{\alpha}+\frac{b_{3} K T^{\alpha+1}}{\Gamma(\alpha+1)} r, \\
\leq & r,
\end{aligned}
$$

which signifies that the operator $F$ maps $B_{r}$ into itself. Assumption $\left(h_{1}\right)$ guarantees that $F$ is continuous.

Now, we will demonstrate that $F$ is compact, that is $F B_{r}$ is relative compact. Let $\Omega$ be a bounded subset of $B_{r}$, then $F(\Omega)$ is bounded in $L_{1}(J, R)$, i.e., condition (i) of Kolmogorov compactness criterion [34] is satisfied. It remain to demonstrate that $(F y)_{h} \rightarrow F y$ in $L_{1}(J, R)$ as $h \rightarrow 0$ for each $y \in B_{r}$.

Let $y \in B_{r}$, then

$$
\begin{aligned}
\left\|(F y)_{h}-(F y)\right\|_{L_{1}} & =\int_{0}^{T}\left|(F y)_{h}(t)-(F y)(t)\right| d t \\
& =\int_{0}^{T}\left|\frac{1}{h} \int_{t}^{t+h}(F y)(\eta) d \eta-(F y)(t)\right| d t \\
& \leq \int_{0}^{T}\left(\frac{1}{h} \int_{t}^{t+h}|(F y)(\eta)-(F y)(t)| d \eta\right) d t
\end{aligned}
$$




$$
\begin{aligned}
\leq & \int_{0}^{T} \frac{1}{h} \int_{t}^{t+h} \mid f\left(\eta, b \eta^{\alpha-1}+I^{\alpha} y(\eta), y(\eta), b \int_{0}^{T} s^{\alpha-1} k(\eta, s) d s+\int_{0}^{T} k(\eta, s) \int_{0}^{s} \frac{(s-\xi)^{\alpha-1}}{\Gamma(\alpha)} y(\xi) d \xi d s\right) \\
& -f\left(t, b t^{\alpha-1}+I^{\alpha} y(t), y(t), b \int_{0}^{T} s^{\alpha-1} k(t, s) d s+\int_{0}^{T} k(t, s) \int_{0}^{s} \frac{(s-\xi)^{\alpha-1}}{\Gamma(\alpha)} y(\xi) d \xi d s\right) \mid d \eta d t
\end{aligned}
$$

from assumptions $\left(h_{1}\right)-\left(h_{2}\right)$ and Theorem 2.2 we get $f \in L_{1}(J, R)$, it follows that [34]

$$
\begin{gathered}
\frac{1}{h} \int_{t}^{t+h} \mid f\left(\eta, b \eta^{\alpha-1}+I^{\alpha} y(\eta), y(\eta), b \int_{0}^{T} s^{\alpha-1} k(\eta, s) d s+\int_{0}^{T} k(\eta, s) \int_{0}^{s} \frac{(s-\xi)^{\alpha-1}}{\Gamma(\alpha)} y(\xi) d \xi d s\right) \\
-f\left(t, b t^{\alpha-1}+I^{\alpha} y(t), y(t), b \int_{0}^{T} s^{\alpha-1} k(t, s) d s+\int_{0}^{T} k(t, s) \int_{0}^{s} \frac{(s-\xi)^{\alpha-1}}{\Gamma(\alpha)} y(\xi) d \xi d s\right) \mid d \eta \\
\rightarrow 0 \quad \text { as } h \rightarrow 0, \text { a.e } t \in(0, T] .
\end{gathered}
$$

Hence, $(F y)_{h} \rightarrow(F y)$ uniformly as $h \rightarrow 0$.

Then, by Theorem 2.1, we have got that $F(\Omega)$ is relative compact, i.e., $F$ is a compact operator.

As a consequence of Schauder's fixed point theorem [37], the operator $F$ has a fixed point in $B_{r}$, which demonstrates the existence of at least one solution $y \in B_{r} \subset L_{1}(J, R)$ of the functional integral equation (3.3), consequently from (3.2) we have $u(t)$ has at least one solution, therefore IFDP (1.1)-(1.2) has at least one solution in $B_{r}$.

Now, for uniqueness of solution to IFDP (1.1)-(1.2). Consider the following assumptions: $\left(\mathbf{h}_{\mathbf{4}}\right)$ there exist constants $k_{i} \geq 0, \quad i=1,2,3$ such that

$$
\left|f\left(t, u_{1}, v_{1}, w_{1}\right)-f\left(t, u_{2}, v_{2}, w_{2}\right)\right| \leq k_{1}\left|u_{1}-u_{2}\right|+k_{2}\left|v_{1}-v_{2}\right|+k_{3}\left|w_{1}-w_{2}\right|,
$$

(h) $f(t, 0,0,0) \in L_{1}(J, R)$

for any $u_{j}, v_{j}, w_{j} \in R, j=1,2$ and $t \in J$.

Theorem 3.2. Assume that the conditions $\left(h_{1}\right),\left(h_{3}\right)-\left(h_{5}\right)$ are satisfied. If

$$
A=\frac{k_{1} T^{\alpha}}{\Gamma(\alpha+1)}+k_{2}+\frac{k_{3} K T^{\alpha+1}}{\Gamma(\alpha+1)}<1
$$

then the IFDP (1.1)-(1.2) has a unique solution $u \in L_{1}(J, R)$.

Proof. From condition $\left(h_{4}\right)$ we can obtain,

$$
|f(t, u, v, w)| \leq|f(t, 0,0,0)|+k_{1}|u|+k_{2}|v|+k_{3}|w|,
$$

this show that the assumptions of Theorem 3.1 are satisfied.

Let $y_{1}, y_{2} \in L_{1}(J, R)$ be two solutions of the functional integral equation (3.3), then

$$
\begin{aligned}
\mid y_{1}(t) & -y_{2}(t)|=| f\left(t, b t^{\alpha-1}+I^{\alpha} y_{1}(t), y_{1}(t), b \int_{0}^{T} s^{\alpha-1} k(t, s) d s+\int_{0}^{T} k(t, s) \int_{0}^{s} \frac{(s-\xi)^{\alpha-1}}{\Gamma(\alpha)} y_{1}(\xi) d \xi d s\right) \\
& -f\left(t, b t^{\alpha-1}+I^{\alpha} y_{2}(t), y_{2}(t), b \int_{0}^{T} s^{\alpha-1} k(t, s) d s+\int_{0}^{T} k(t, s) \int_{0}^{s} \frac{(s-\xi)^{\alpha-1}}{\Gamma(\alpha)} y_{2}(\xi) d \xi d s\right) \mid, \\
\leq & k_{1} \int_{0}^{t} \frac{(t-s)^{\alpha-1}}{\Gamma(\alpha)}\left|y_{1}(s)-y_{2}(s)\right| d s+k_{2}\left|y_{1}(t)-y_{2}(t)\right| \\
& \quad+k_{3} K \int_{0}^{T} \int_{0}^{s} \frac{(s-\xi)^{\alpha-1}}{\Gamma(\alpha)}\left|y_{1}(\xi)-y_{2}(\xi)\right| d \xi d s, \\
\leq & k_{1} \int_{0}^{t} \frac{(t-s)^{\alpha-1}}{\Gamma(\alpha)}\left|y_{1}(s)-y_{2}(s)\right| d s+k_{2}\left|y_{1}(t)-y_{2}(t)\right|+k_{3} K \int_{0}^{T} \frac{(T-\xi)^{\alpha}}{\Gamma(\alpha+1)}\left|y_{1}(\xi)-y_{2}(\xi)\right| d \xi, \\
\leq & k_{1} \int_{0}^{t} \frac{(t-s)^{\alpha-1}}{\Gamma(\alpha)}\left|y_{1}(s)-y_{2}(s)\right| d s+k_{2}\left|y_{1}(t)-y_{2}(t)\right|+\frac{k_{3} K T^{\alpha}}{\Gamma(\alpha+1)}|| y_{1}-y_{2}||_{L_{1}} .
\end{aligned}
$$


Thus

$$
\begin{aligned}
\left\|y_{1}-y_{2}\right\|_{L_{1}} \leq & k_{1} \int_{0}^{T} \int_{0}^{t} \frac{(t-s)^{\alpha-1}}{\Gamma(\alpha)}\left|y_{1}(s)-y_{2}(s)\right| d s d t+k_{2} \int_{0}^{T}\left|y_{1}(t)-y_{2}(t)\right| d t \\
& +\frac{k_{3} K T^{\alpha+1}}{\Gamma(\alpha+1)}\left\|y_{1}-y_{2}\right\|_{L_{1}}, \\
\leq & \left.k_{1} \int_{0}^{T} \mid y_{1}(s)\right)-y_{2}(s) \mid d s \int_{s}^{T} \frac{(t-s)^{\alpha-1}}{\Gamma(\alpha)} d t+k_{2}\left\|y_{1}-y_{2}\right\|_{L_{1}} \\
& +\frac{k_{3} K T^{\alpha+1}}{\Gamma(\alpha+1)}\left\|y_{1}-y_{2}\right\|_{L_{1}}, \\
\leq & \left(\frac{k_{1} T^{\alpha}}{\Gamma(\alpha+1)}+k_{2}+\frac{k_{3} K T^{\alpha+1}}{\Gamma(\alpha+1)}\right)\left\|y_{1}-y_{2}\right\|_{L_{1}} \leq A\left\|y_{1}-y_{2}\right\|_{L_{1}},
\end{aligned}
$$

then, $\quad(1-A)\left\|y_{1}-y_{2}\right\|_{L_{1}} \leq 0$ which signifies that $\left\|y_{1}-y_{2}\right\|_{L_{1}}=0$, and we have $y_{1}=y_{2}$, then there exists a unique integrable solution of the problem (1.1)-(1.2).

Example 3.1. Consider the following IFDP:

$$
\begin{gathered}
D^{\frac{1}{2}} u(t)=h(t)\left(\frac{(t-3)^{2}}{1+\left(\int_{0}^{1} e^{t-s} u(s) d s\right)^{2}}+\frac{\cos t}{10} \frac{u^{2}(t)+e^{-t}\left(D^{\frac{1}{2}} u\right)(t)}{1+|u(t)|+\left|\left(D^{\frac{1}{2}} u\right)(t)\right|}+\frac{1}{6} \sin \left(\int_{0}^{1} e^{t-s} u(s) d s\right)\right) \text {, a.e. } t \in(0,1], \\
\left.I^{\frac{1}{2}} u(t)\right|_{t=0}=b \Gamma\left(\frac{1}{2}\right), \quad b \in R .
\end{gathered}
$$

where $h(t)=\left\{\begin{array}{cc}t, & 0<t \leq \frac{1}{2}, \\ t^{2}, & \frac{1}{2}<t \leq 1 .\end{array}\right.$

Set $f(t, u, v, w)=h(t)\left(\frac{(t-3)^{2}}{1+w^{2}}+\frac{\cos t}{10} \frac{u^{2}+e^{-t} v}{1+|u|+|v|}+\frac{1}{6} \sin w\right)$, then we have

$$
\begin{aligned}
|f(t, u, v, w)| & \leq(t-3)^{2}+\frac{1}{10}\left(\frac{\left|u^{2}\right|}{1+|u|+|v|}+\frac{e^{-t}|v|}{1+|u|+|v|}\right)+\frac{1}{6}|\sin w| \\
& \leq(t-3)^{2}+\frac{1}{10}(|u|+|v|)+\frac{1}{6}|w|,
\end{aligned}
$$

and the condition $\frac{b_{1} T^{\alpha}}{\Gamma(\alpha+1)}+b_{2}+\frac{b_{3} K T^{\alpha+1}}{\Gamma(\alpha+1)}=0.72263935<1$, is satisfied with $\alpha=\frac{1}{2}, b_{1}=\frac{1}{10}, b_{2}=\frac{1}{10}, b_{3}=\frac{1}{6}, K=e, T=1$. It follows from Theorem 3.1 that the problem has at least one integrable solution on $(0,1]$.

Example 3.2. Consider the following IFDP:

$$
\begin{gathered}
D^{\frac{1}{5}} u(t)=\frac{e^{-t}}{1+4 e^{t}} \frac{1}{1+\int_{0}^{1} \ln (t+s) u(s) d s|+| u(t) \mid}+\frac{1}{10} \cos \left(D^{\frac{1}{5}} u(t)\right), \quad \text { a.e. } t \in(0,1], \\
\left.I^{\frac{4}{5}} u(t)\right|_{t=0}=b \Gamma\left(\frac{1}{5}\right), \quad b \in R .
\end{gathered}
$$

Set $f(t, u, v, w)=\frac{e^{-t}}{1+4 e^{t}} \frac{1}{1+|w|+|u|}+\frac{1}{10} \cos v$, then we have

$$
\begin{aligned}
\left|f\left(t, u_{1}, v_{1}, w_{1}\right)-f\left(t, u_{2}, v_{2}, w_{2}\right)\right| \leq & \frac{e^{-t}}{1+4 e^{t}}\left|\frac{1}{1+\left|w_{1}\right|+\left|u_{1}\right|}-\frac{1}{1+\left|w_{2}\right|+\left|u_{2}\right|}\right| \\
& +\frac{1}{10}\left|\cos v_{1}-\cos v_{2}\right|, \\
\leq & \frac{1}{5} \frac{\left|u_{1}-u_{2}\right|+\left|w_{1}-w_{2}\right|}{\left(1+\left|w_{1}\right|+\left|u_{1}\right|\right)\left(1+\left|w_{2}\right|+\left|u_{2}\right|\right)}+\frac{1}{10}\left|v_{1}-v_{2}\right|, \\
\leq & \frac{1}{5}\left(\left|u_{1}-u_{2}\right|+\left|w_{1}-w_{2}\right|\right)+\frac{1}{10}\left|v_{1}-v_{2}\right|,
\end{aligned}
$$

and $f(t, 0,0,0)=\frac{e^{-t}}{1+4 e^{t}}+\frac{1}{10} \in L_{1}(0,1]$, also $\frac{k_{1} T^{\alpha}}{\Gamma(\alpha+1)}+k_{2}+\frac{k_{3} K T^{\alpha+1}}{\Gamma(\alpha+1)}=0.4688095<1$ is satisfied with $\alpha=\frac{1}{5}, k_{1}=\frac{1}{5}, k_{2}=$ $\frac{1}{10}, k_{3}=\frac{1}{5}, K=\ln 2, T=1$. It follows from Theorem 3.2 that the problem has a unique integrable solution in $(0,1]$. 


\section{Unique solution in the weighted space $C_{1-\alpha}(\bar{J}, R)$}

Here we examine the existence of a unique solution of IFDP (1.1)-(1.2) in the weighted space $C_{1-\alpha}(\bar{J}, R)$.

Definition 4.1. By a solution of IFDP (1.1)-(1.2) we mean a function $\left\{u: t^{1-\alpha} u(t)\right.$ is continuous on the interval $\left.\bar{J}\right\}$ and this function satisfies (1.1)-(1.2).

Consider the following assumption:

$\left(\mathbf{h}_{\mathbf{6}}\right) f: \bar{J} \times R \times R \times R \rightarrow R$ is continuous function with $\max _{t \in \bar{J}}|f(t, 0,0,0)| \leq f_{0}$.

Theorem 4.1. Let the assumptions $\left(h_{3}\right),\left(h_{4}\right)$ and $\left(h_{6}\right)$ be satisfied. If

$$
\frac{\left(2 \alpha k_{1}+k_{3} K T^{\alpha}\right) \Gamma(\alpha) T^{\alpha}}{\Gamma(2 \alpha+1)}+k_{2}<1
$$

then IFDP (1.1)-(1.2) has a unique solution $u \in C_{1-\alpha}(\bar{J}, R)$.

Proof. Define the operator $F$ by (3.6), the operator $F$ maps $C_{1-\alpha}(\bar{J}, R)$ into it self, for this let $y \in C_{1-\alpha}(\bar{J}, R), t_{1}, t_{2} \in$ $\bar{J}, t_{1}<t_{2}$ such that $\left|t_{2}-t_{1}\right|<\delta$, we have

$$
\begin{aligned}
& \left|t_{2}^{1-\alpha}(F y)\left(t_{2}\right)-t_{1}^{1-\alpha}(F y)\left(t_{1}\right)\right| \\
= & \mid t_{2}^{1-\alpha} f\left(t_{2}, b t_{2}^{\alpha-1}+I^{\alpha} y\left(t_{2}\right), y\left(t_{2}\right), b \int_{0}^{T} s^{\alpha-1} k\left(t_{2}, s\right) d s+\int_{0}^{T} k\left(t_{2}, s\right) \int_{0}^{s} \frac{(s-\xi)^{\alpha-1}}{\Gamma(\alpha)} y(\xi) d \xi d s\right) \\
- & t_{1}^{1-\alpha} f\left(t_{2}, b t_{2}^{\alpha-1}+I^{\alpha} y\left(t_{2}\right), y\left(t_{2}\right), b \int_{0}^{T} s^{\alpha-1} k\left(t_{2}, s\right) d s+\int_{0}^{T} k\left(t_{2}, s\right) \int_{0}^{s} \frac{(s-\xi)^{\alpha-1}}{\Gamma(\alpha)} y(\xi) d \xi d s\right) \\
+ & t_{1}^{1-\alpha} f\left(t_{2}, b t_{2}^{\alpha-1}+I^{\alpha} y\left(t_{2}\right), y\left(t_{2}\right), b \int_{0}^{T} s^{\alpha-1} k\left(t_{2}, s\right) d s+\int_{0}^{T} k\left(t_{2}, s\right) \int_{0}^{s} \frac{(s-\xi)^{\alpha-1}}{\Gamma(\alpha)} y(\xi) d \xi d s\right) \\
- & t_{1}^{1-\alpha} f\left(t_{1}, b t_{1}^{\alpha-1}+I^{\alpha} y\left(t_{1}\right), y\left(t_{1}\right), b \int_{0}^{T} s^{\alpha-1} k\left(t_{1}, s\right) d s+\int_{0}^{T} k\left(t_{1}, s\right) \int_{0}^{s} \frac{(s-\xi)^{\alpha-1}}{\Gamma(\alpha)} y(\xi) d \xi d s\right) \mid \\
\leq & \left|t_{2}^{1-\alpha}-t_{1}^{1-\alpha}\right| \\
& \times\left|f\left(t_{2}, b t_{2}^{\alpha-1}+I^{\alpha} y\left(t_{2}\right), y\left(t_{2}\right), b \int_{0}^{T} s^{\alpha-1} k\left(t_{2}, s\right) d s+\int_{0}^{T} k\left(t_{2}, s\right) \int_{0}^{s} \frac{(s-\xi)^{\alpha-1}}{\Gamma(\alpha)} y(\xi) d \xi d s\right)\right| \\
+ & t_{1}^{1-\alpha} \mid f\left(t_{2}, b t_{2}^{\alpha-1}+I^{\alpha} y\left(t_{2}\right), y\left(t_{2}\right), b \int_{0}^{T} s^{\alpha-1} k\left(t_{2}, s\right) d s+\int_{0}^{T} k\left(t_{2}, s\right) \int_{0}^{s} \frac{(s-\xi)^{\alpha-1}}{\Gamma(\alpha)} y(\xi) d \xi d s\right) \\
& -f\left(t_{2}, b t_{1}^{\alpha-1}+I^{\alpha} y\left(t_{1}\right), y\left(t_{1}\right), b \int_{0}^{T} s^{\alpha-1} k\left(t_{1}, s\right) d s+\int_{0}^{T} k\left(t_{1}, s\right) \int_{0}^{s} \frac{(s-\xi)^{\alpha-1}}{\Gamma(\alpha)} y(\xi) d \xi d s\right) \mid \\
& +t_{1}^{1-\alpha} \mid f\left(t_{2}, b t_{1}^{\alpha-1}+I^{\alpha} y\left(t_{1}\right), y\left(t_{1}\right), b \int_{0}^{T-1} s^{\alpha-1} k\left(t_{1}, s\right) d s+I_{0}^{\alpha} y\left(t_{1}\right), y\left(t_{1}\right), b \int_{0}^{T} s^{\alpha-1} k\left(t_{1}, s\right) d s+\int_{0}^{T} k\left(t_{1}, s\right) \int_{0}^{s} \frac{(s-\xi)^{\alpha-1}}{\Gamma(\alpha)} y(\xi) d \xi d s\right) \\
& \quad \mid f(\xi) d \xi d s) \mid .
\end{aligned}
$$

Firstly, we evaluate $f$ in the first term of the last inequality, from condition $\left(h_{4}\right)$ we have

$$
\left|f\left(t_{2}, b t_{2}^{\alpha-1}+I^{\alpha} y\left(t_{2}\right), y\left(t_{2}\right), b \int_{0}^{T} s^{\alpha-1} k\left(t_{2}, s\right) d s+\int_{0}^{T} k\left(t_{2}, s\right) \int_{0}^{s} \frac{(s-\xi)^{\alpha-1}}{\Gamma(\alpha)} y(\xi) d \xi d s\right)\right|
$$




$$
\begin{aligned}
\leq & \left|f\left(t_{2}, 0,0,0\right)\right|+\mid f\left(t_{2}, b t_{2}^{\alpha-1}+I^{\alpha} y\left(t_{2}\right), y\left(t_{2}\right), b \int_{0}^{T} s^{\alpha-1} k\left(t_{2}, s\right) d s\right. \\
& \left.\quad+\int_{0}^{T} k\left(t_{2}, s\right) \int_{0}^{s} \frac{(s-\xi)^{\alpha-1}}{\Gamma(\alpha)} y(\xi) d \xi d s\right)-f\left(t_{2}, 0,0,0\right) \mid \\
\leq & f_{0}+k_{1}|b| t_{2}^{\alpha-1}+k_{1} \int_{0}^{t_{2}} \frac{\left(t_{2}-s\right)^{\alpha-1}}{\Gamma(\alpha)}|y(s)| d s+k_{2} t_{2}^{\alpha-1} t_{2}^{1-\alpha}\left|y\left(t_{2}\right)\right| \\
& +k_{3}|b| \int_{0}^{T} s^{\alpha-1}\left|k\left(t_{2}, s\right)\right| d s+k_{3} \int_{0}^{T}\left|k\left(t_{2}, s\right)\right| \int_{0}^{s} \frac{(s-\xi)^{\alpha-1}}{\Gamma(\alpha)} \xi^{\alpha-1} \xi^{1-\alpha}|y(\xi)| d \xi d s, \\
\leq & f_{0}+k_{1}|b| t_{2}^{\alpha-1}+k_{1}|| y\left\|_{C_{1-\alpha}} \int_{0}^{t_{2}} \frac{\left(t_{2}-s\right)^{\alpha-1}}{\Gamma(\alpha)} s^{\alpha-1} d s+k_{2} t_{2}^{\alpha-1}|| y\right\|_{C_{1-\alpha}} \\
& +k_{3}|b| K \int_{0}^{T} s^{\alpha-1} d s+k_{3} K\|y\|_{C_{1-\alpha}} \int_{0}^{T} d s \int_{0}^{s} \frac{(s-\xi)^{\alpha-1}}{\Gamma(\alpha)} \xi^{\alpha-1} d \xi \\
\leq & f_{0}+k_{1}|b| t_{2}^{\alpha-1}+\frac{k_{1} T^{2 \alpha-1} \Gamma(\alpha)}{\Gamma(2 \alpha)}\|y\|_{C_{1-\alpha}}+k_{2} t_{2}^{\alpha-1}\|y\|_{C_{1-\alpha}}+\frac{k_{3}|b| K T^{\alpha}}{\alpha} \\
& +\frac{k_{3} K T^{2 \alpha} \Gamma(\alpha)}{\Gamma(2 \alpha+1)}\|y\|_{C_{1-\alpha}} .
\end{aligned}
$$

Secondly, we evaluate the second term in the inequality (4.1), we have

$$
\begin{gathered}
\mid f\left(t_{2}, b t_{2}^{\alpha-1}+I^{\alpha} y\left(t_{2}\right), y\left(t_{2}\right), b \int_{0}^{T} s^{\alpha-1} k\left(t_{2}, s\right) d s+\int_{0}^{T} k\left(t_{2}, s\right) \int_{0}^{s} \frac{(s-\xi)^{\alpha-1}}{\Gamma(\alpha)} y(\xi) d \xi d s\right) \\
-f\left(t_{2}, b t_{1}^{\alpha-1}+I^{\alpha} y\left(t_{1}\right), y\left(t_{1}\right), b \int_{0}^{T} s^{\alpha-1} k\left(t_{1}, s\right) d s+\int_{0}^{T} k\left(t_{1}, s\right) \int_{0}^{s} \frac{(s-\xi)^{\alpha-1}}{\Gamma(\alpha)} y(\xi) d \xi d s\right) \mid \\
\leq k_{1}|b|\left|t_{2}^{\alpha-1}-t_{1}^{\alpha-1}\right|+k_{1} \int_{0}^{t_{1}}\left|\frac{\left(t_{2}-s\right)^{\alpha-1}}{\Gamma(\alpha)}-\frac{\left(t_{1}-s\right)^{\alpha-1}}{\Gamma(\alpha)}\right| y(s) \mid d s \\
\quad+k_{1} \int_{t_{1}}^{t_{2}} \frac{\left(t_{2}-s\right)^{\alpha-1}}{\Gamma(\alpha)}|y(s)| d s+k_{2}\left|y\left(t_{2}\right)-y\left(t_{1}\right)\right|+k_{3}|b| \int_{0}^{T} s^{\alpha-1}\left|k\left(t_{2}, s\right)-k\left(t_{1}, s\right)\right| d s \\
\quad+k_{3} \int_{0}^{T}\left|k\left(t_{2}, s\right)-k\left(t_{1}, s\right)\right| \int_{0}^{s} \frac{(s-\xi)^{\alpha-1}}{\Gamma(\alpha)}|y(\xi)| d \xi d s, \\
\leq k_{1}|b|\left|t_{2}^{\alpha-1}-t_{1}^{\alpha-1}\right|+k_{1} \int_{0}^{t_{1}}\left|\frac{\left(t_{2}-s\right)^{\alpha-1}}{\Gamma(\alpha)}-\frac{\left(t_{1}-s\right)^{\alpha-1}}{\Gamma(\alpha)}\right| s^{\alpha-1} s^{1-\alpha}|y(s)| d s \\
+k_{1} \int_{t_{1}}^{t_{2}} \frac{\left(t_{2}-s\right)^{\alpha-1}}{\Gamma(\alpha)} s^{\alpha-1} s^{1-\alpha}|y(s)| d s \\
+k_{2}\left|t_{2}^{\alpha-1} t_{2}^{1-\alpha} y\left(t_{2}\right)-t_{1}^{\alpha-1} t_{2}^{1-\alpha} y\left(t_{2}\right)-t_{1}^{\alpha-1} t_{2}^{1-\alpha} y\left(t_{2}\right)-t_{1}^{\alpha-1} t_{1}^{1-\alpha} y\left(t_{1}\right)\right| \\
+k_{3}|b| \int_{0}^{T} s^{\alpha-1}\left|k\left(t_{2}, s\right)-k\left(t_{1}, s\right)\right| d s+k_{3} \int_{0}^{T}\left|k\left(t_{2}, s\right)-k\left(t_{1}, s\right)\right| \int_{0}^{s} \frac{(s-\xi)^{\alpha-1}}{\Gamma(\alpha)} \xi^{\alpha-1} \xi^{1-\alpha}|y(\xi)| d \xi d s,
\end{gathered}
$$




$$
\begin{aligned}
& \leq k_{1}|b|\left|t_{2}^{\alpha-1}-t_{1}^{\alpha-1}\right|+\left.k_{1}|| y\right|_{C_{1-\alpha}} \int_{t_{1}}^{t_{2}} \frac{\left(t_{2}-s\right)^{\alpha-1}}{\Gamma(\alpha)} s^{\alpha-1} d s \\
& \quad+\left.k_{1}|| y\right|_{C_{1-\alpha}} \int_{0}^{t_{1}}\left(\frac{\left(t_{1}-s\right)^{\alpha-1}}{\Gamma(\alpha)}-\frac{\left(t_{2}-s\right)^{\alpha-1}}{\Gamma(\alpha)}\right) s^{\alpha-1} d s+k_{2}\left|t_{2}^{\alpha-1}-t_{1}^{\alpha-1}\right| t_{2}^{1-\alpha}\left|y\left(t_{2}\right)\right| \\
& \quad+k_{2} t_{1}^{\alpha-1}\left|t_{2}^{1-\alpha} y\left(t_{2}\right)-t_{1}^{1-\alpha} y\left(t_{1}\right)\right|+k_{3}|b| \int_{0}^{T} s^{\alpha-1}\left|k\left(t_{2}, s\right)-k\left(t_{1}, s\right)\right| d s \\
& +\frac{k_{3} T^{2 \alpha-1} \Gamma(\alpha)|| y \|_{C_{1-\alpha}}}{\Gamma(2 \alpha)} \int_{0}^{T}\left|k\left(t_{2}, s\right)-k\left(t_{1}, s\right)\right| d s \\
& \leq k_{1}|b|\left|t_{2}^{\alpha-1}-t_{1}^{\alpha-1}\right|+k_{1} t_{2}^{2 \alpha-1}|| y||_{C_{1-\alpha}} \int_{\frac{t_{1}}{t_{2}}}^{1} \frac{(1-\xi)^{\alpha-1} \xi^{\alpha-1}}{\Gamma(\alpha)} d \xi \\
& +k_{1}|| y \|_{C_{1-\alpha}}\left[\frac{t_{1}^{2 \alpha-1} \Gamma(\alpha)}{\Gamma(2 \alpha)}-\frac{t_{2}^{2 \alpha-1}}{\Gamma(\alpha)} \int_{0}^{\frac{t_{t}}{t_{2}}}(1-\xi)^{\alpha-1} \xi^{\alpha-1} d \xi\right] \\
& +k_{2}\left|t_{2}^{\alpha-1}-t_{1}^{\alpha-1}\right|\|y\|_{C_{1-\alpha}}+k_{2} t_{1}^{\alpha-1}\left|t_{2}^{1-\alpha} y\left(t_{2}\right)-t_{1}^{1-\alpha} y\left(t_{1}\right)\right| \\
& \quad+k_{3}|b| \int_{0}^{T} s^{\alpha-1}\left|k\left(t_{2}, s\right)-k\left(t_{1}, s\right)\right| d s+\frac{k_{3} T^{2 \alpha-1} \Gamma(\alpha)|| y \|_{C_{1-\alpha}}}{\Gamma(2 \alpha)} \int_{0}^{T}\left|k\left(t_{2}, s\right)-k\left(t_{1}, s\right)\right| d s .
\end{aligned}
$$

Now we return to equation (4.1), substituting from (4.2) and (4.3) into (4.1), we obtain

$$
\begin{gathered}
\left|t_{2}^{1-\alpha}(F y)\left(t_{2}\right)-t_{1}^{1-\alpha}(F y)\left(t_{1}\right)\right| \\
\left|t_{2}^{\alpha-1}-t_{1}^{\alpha-1}\right|\left(f_{0}+k_{1}|b| t_{2}^{\alpha-1}+\frac{k_{1} T^{2 \alpha-1} \Gamma(\alpha)}{\Gamma(2 \alpha)}\|y\|_{C_{1-\alpha}}+k_{2} t_{2}^{\alpha-1}|| y \|_{C_{1-\alpha}}+\frac{k_{3}|b| K T^{\alpha}}{\alpha}\right. \\
\left.+\frac{k_{3} K T^{2 \alpha} \Gamma(\alpha)}{\Gamma(2 \alpha+1)} \|\left. y\right|_{C_{1-\alpha}}\right)+k_{1}|b|\left|t_{2}^{\alpha-1}-t_{1}^{\alpha-1}\right|+k_{1} t_{2}^{2 \alpha-1}|| y \|_{C_{1-\alpha}} \int_{\frac{t_{1}}{t_{2}}}^{1} \frac{(1-\xi)^{\alpha-1} \xi^{\alpha-1}}{\Gamma(\alpha)} d \xi \\
+k_{1}|| y \|_{C_{1-\alpha}}\left[\frac{t_{1}^{2 \alpha-1} \Gamma(\alpha)}{\Gamma(2 \alpha)}-\frac{t_{2}^{2 \alpha-1}}{\Gamma(\alpha)} \int_{0}^{\frac{t_{1}}{t_{2}}}(1-\xi)^{\alpha-1} \xi^{\alpha-1} d \xi\right] \\
+k_{2}\left|t_{2}^{\alpha-1}-t_{1}^{\alpha-1}\right||| y \|_{C_{1-\alpha}}+k_{2} t_{1}^{\alpha-1}\left|t_{2}^{1-\alpha} y\left(t_{2}\right)-t_{1}^{1-\alpha} y\left(t_{1}\right)\right| \\
+k_{3}|b| \int_{0}^{T} s^{\alpha-1}\left|k\left(t_{2}, s\right)-k\left(t_{1}, s\right)\right| d s+\frac{k_{3} T^{2 \alpha-1} \Gamma(\alpha)\|y\|_{C_{1-\alpha}}}{\Gamma(2 \alpha)} \int_{0}^{T}\left|k\left(t_{2}, s\right)-k\left(t_{1}, s\right)\right| d s . \\
+t_{1}^{1-\alpha} \mid f\left(t_{2}, b t_{1}^{\alpha-1}+I^{\alpha} y\left(t_{1}\right), y\left(t_{1}\right), b \int_{0}^{T} s^{\alpha-1} k\left(t_{1}, s\right) d s+\int_{0}^{T} k\left(t_{1}, s\right) \int_{0}^{s} \frac{(s-\xi)^{\alpha-1}}{\Gamma(\alpha)} y(\xi) d \xi d s\right) \\
-f\left(t_{1}, b t_{1}^{\alpha-1}+I^{\alpha} y\left(t_{1}\right), y\left(t_{1}\right), b \int_{0}^{T} s^{\alpha-1} k\left(t_{1}, s\right) d s+\int_{0}^{T} k\left(t_{1}, s\right) \int_{0}^{s} \frac{(s-\xi)^{\alpha-1}}{\Gamma(\alpha)} y(\xi) d \xi d s\right) \mid .
\end{gathered}
$$

As $t_{2} \rightarrow t_{1}$, the right side of the above inequality tends to zero.

And therefore the operator $F: C_{1-\alpha}(\bar{J}, R) \rightarrow C_{1-\alpha}(\bar{J}, R)$. 
Now to prove $F$ is a contraction mapping, let $y, z \in C_{1-\alpha}(\bar{J}, R)$, then

$$
\begin{aligned}
& \left|t^{1-\alpha}(F y)(t)-t^{1-\alpha}(F z)(t)\right| \\
& =t^{1-\alpha} \mid f\left(t, b t^{\alpha-1}+I^{\alpha} y(t), y(t), b \int_{0}^{T} s^{\alpha-1} k(t, s) d s+\int_{0}^{T} k(t, s) \int_{0}^{s} \frac{(s-\xi)^{\alpha-1}}{\Gamma(\alpha)} y(\xi) d \xi d s\right) \\
& \quad-f\left(t, b t^{\alpha-1}+I^{\alpha} z(t), z(t), b \int_{0}^{T} s^{\alpha-1} k(t, s) d s+\int_{0}^{T} k(t, s) \int_{0}^{s} \frac{(s-\xi)^{\alpha-1}}{\Gamma(\alpha)} z(\xi) d \xi d s\right) \mid \\
& \leq k_{1} t^{1-\alpha} \int_{0}^{t} \frac{(t-s)^{\alpha-1}}{\Gamma(\alpha)}|y(s)-z(s)| d s+k_{2} t^{1-\alpha}|y(t)-z(t)| \\
& \quad+k_{3} t^{1-\alpha} \int_{0}^{T}|k(t, s)| \int_{0}^{s} \frac{(s-\xi)^{\alpha-1}}{\Gamma(\alpha)}|y(\xi)-z(\xi)| d \xi d s \\
& \leq k_{1} T^{1-\alpha}|| y-z\left\|_{C_{1-\alpha}} \int_{0}^{t} \frac{(t-s)^{\alpha-1}}{\Gamma(\alpha)} s^{\alpha-1} d s+k_{2}\right\| y-z \|_{C_{1-\alpha}} \\
& \quad+k_{3} K T^{1-\alpha}\|y-z\|_{C_{1-\alpha}} \int_{0}^{T} \int_{0}^{s} \frac{(s-\xi)^{\alpha-1}}{\Gamma(\alpha)} \xi^{\alpha-1} d \xi d s \\
& \leq\left[\frac{k_{1} \Gamma(\alpha) T^{\alpha}}{\Gamma(2 \alpha)}+k_{2}+\frac{k_{3} K \Gamma(\alpha) T^{2 \alpha}}{\Gamma(2 \alpha+1)}\right]\|y-z\|_{C_{1-\alpha}},
\end{aligned}
$$

this implies that

$$
\|F y-F z\|_{C_{1-\alpha}} \leq\left[\frac{\left(2 \alpha k_{1}+k_{3} K T^{\alpha}\right) \Gamma(\alpha) T^{\alpha}}{\Gamma(2 \alpha+1)}+k_{2}\right]\|y-z\|_{C_{1-\alpha}} .
$$

If $\frac{\left(2 \alpha k_{1}+k_{3} K T^{\alpha}\right) \Gamma(\alpha) T^{\alpha}}{\Gamma(2 \alpha+1)}+k_{2}<1$, then $F$ is a contraction mapping and by using "Banach Contraction Principle", there exists a unique solution $y \in C_{1-\alpha}(\bar{J}, R)$ of the functional integral equation (3.3), and consequently there exists a unique solution of IFDP (1.1)-(1.2).

Acknowledgements. The author wishes to express here thanks to the referees for a careful reading of this paper and the valuable comments and suggestions.

\section{References}

[1] D. Baleanu, K. Diethelm, E. Scalas and J. J. Trujillo, Fractional calculus. models and numerical methods. Series on Complexity, Nonlinearity and Chaos 3. World Scientific, Hackensack, NJ, 2012.

[2] C. F. M. Coimbra, C. M. Soon, M. H. Kobayashi, The variable viscoelasticity operator, Annalen der Physik, 14 (2005) 378-389.

[3] C. F. M. Coimbra, Mechanics with variable order differential operators, Annalen der Physik, 12(11-12) (2003) 692-703.

[4] M. Dalir, M. Bashour, Applications of fractional calculus, Appl Math Sci, 4(21) (2010) 1021-1032.

[5] K. Diethelm, The analysis of fractional differential equations, Lecture Notes in Mathematics, 2004 Springer-Verlag, Berlin (2010).

[6] W.G. Glockle, T.F. Nonnenmacher, A fractional calculus approach of self-similar protein dynamics, Biophys. J, 68 (1995) 46-53.

[7] R. Hilfer(Editor), Applications of fractional calculus in physics, Word Scientific Publishing Co., New Jersey, London, Hong Kong, 2000.

[8] D. Ingman, J. Suzdalnitsky, Control of damping oscillations by fractional differential operator with time-dependent order, Comput Meth Appl Mech Eng, 193(52) (2004) 5585-5595. 
[9] A.A. Kilbas, H.M. Srivastava, J. J. Trujillo, Theory and applications of fractional differential equations, Elsevier, Amsterdam, (2006).

[10] J. A. T. Machado, M. F. Silva, R. S. Barbosa, I. S. Jesus, C. M. Reis, et al., Some applications of fractional calculus in engineering, Math Probl Eng 34 (2010) Art. ID 639801.

[11] F. Metzler, W. Schick, H.G. Kilian and T.F. Nonnenmacher, Relaxation in filled polymers: a fractional calculus approach, J. Chem. Phys. vol. 103 (1995) 7180-7186.

[12] Y.A. Rossikhin, M.V. Shitikova, Application of fractional calculus for dynamic problems of solid mechanics: novel trends and recent results, Appl. Mech. Rev. 63 (1) (2010) 010801-1-51.

[13] J. Sabatier, O. P. Agrawal, J.A. Tenreiro Machado, Advances in fractional calculus: Theoretical Developments and Applications in Physics and Engineering, Springer-Verlag, Berlin, (2007).

[14] S. Samko, O. L. Marichev, Fractional integral and derivatives, Gordon and Breach science Publisher, (1993).

[15] N. H. Sweilam, H. M. AL-Mrawm, On thenumerical solutions of the variable order fractional heat equation, Stud. Nonlinear Sci, 2 (2011) 31-36.

[16] T. Yajima, K. Yamasaki, Geometry of surfaces with Caputo fractional derivatives and applications to incompressible two-dimensional flows, J Phys A 45(6) (2012) DOI: 10.1088/1751-8113/45/6/065201.

[17] S. Abbas, M. Benchohra and A. N. Vityuk, On fractional order derivatives and Darboux problem for implicit differential equations, Frac. Calc. Appl. Anal. 15(2) (2012) 168-182.

[18] T. D. Benavides, An existence theorem for implicit differential equations in a Banach space, Ann. Mat. Pura Appl. 4 (1978) 119-130.

[19] M. Benchohra and M. Souid, Integrable solutions for implicit fractional order differential equations, TJMM, 6(2) (2014) 101-107.

[20] M. Benchohra and M. Souid, $L^{1}$-solutions of boundary value problems for implict fractional order differential equations, Surv. Math. Appl., 10 (2015) 49-59.

[21] M. Benchohra and J. E. Lazreg, Existence and Ulam stability for nonlinear implicit fractional differential equations with Hadamard derivative, Stud. Univ. Babes-Bolyai Math. 62(1) (2017) 27-38.

[22] M. Benchohra and M. Souid, Integrable solutions for implicit fractional order functional differential equations with infinite delay, Archivum Mathematicum (Brno) Tomus, 51 (2015) 67-76.

[23] M. Benchohra and M. S. Souid and Integrable solutions for implicit fractional order functional differential equations with infinite delay, Archivum Mathematicum (BRNO) Tomus, 51 (2015), 67-76.

[24] A. M. A. El-Sayed and E. O. Bin-Taher, Positive solutions for a nonlocal boundary-value problem of a class of arbitrary (fractional) orders differential equations, Intern. J. nonlinear Sci, 14(4) (2012) 398-404.

[25] A. M. A. El-Sayed and E. O. Bin-Taher, Nonlocal and integrable conditions problems for a multi-term fractional-order differential equations, Miskolc Math. Notes, 15(2) (2014) 439-446.

[26] A. M. A. El-Sayed and E. O. Bin-Taher, Positive solutions for a nonlocal multi-point bounday-value problem of fractional and second order, Electronic J. Differ. Equ., 2013(64) (2014) 1-8.

[27] A. Guezane-Lakoud and R. Khaldi, Solvability of a fractional boundary value problem with fractional integral condition, Nonlinear Anal. 75 (2012) 2692-2700.

[28] J. J. Nieto, A. Ouahab and V. Venktesh, Implicit fractional differential equations via the Liouville-Caputo derivative, Mathematics 3 (2015), 398-411.

[29] A. N. Vityuk and A. V. Mykhailenko, The Darboux problem for an implicit fractional-order differential equation, J. Math. Sci., 175 (4) (2011), 391-401. 
[30] A.M.A. El-Sayed and Sh. A. Abd El-salam, Weighted Cauchy-type problem of a functional differ-integral equation, EJQTDE, 2007 (30) (2007) 1-9.

[31] A. M. A. El-Sayed and Sh. A. Abd El-Salam, $L^{p}$-solution of weighted Cauchy-type problem of a diffre-integral functional equation, Intern. J. Nonlinear Sci., 5 (2008) 281-288.

[32] A.M.A. El-Sayed and Sh. A. Abd El-salam, Nonlocal boundary value problem of a fractional-order functional differential equation, Intern. J. Nonliear Sci., 7(4) (2009), 436-442.

[33] A.M.M. El-Sayed, H.H.G. Hashem, Integrable and continuous solutions of a nonlinear quadratic integral equation, Electron. J. Qual. Theory Differ. Equ., 25(2008) 1-10

[34] C. Swartz, Measure, Integration and functional spaces, World Scientific, Singapore (1994).

[35] J. Appell, P. P. Zabrejko, Nonlinear superposition 0perators, Cambridge Tracts in Mathematics, Vol. 95, Cambridge University Press, Cambridge 1990; Zbl. 701.47041; MR 91k:47168.

[36] M. A. Krasnosel'skii, On the continuity of the operator $F u(x)=f(x, u(x))$, Dokl. Akad. Nauk SSSR, 77 (1951), 185-188 [in Russian].

[37] K. Deimling, Nonlinear Functional analysis, Springer-verlag (1985). 